\title{
Predictors of Maternal Identity of Korean Primiparas
}

\author{
Chae, Hyun-Ju' ${ }^{1}$ Song, Ju-Eun ${ }^{2} \cdot \mathrm{Kim}_{\mathrm{S}} \mathrm{Sue}^{3}$ \\ ${ }^{1}$ Part-time Lecturer, College of Nursing, Sungshin Women's University, Seoul \\ ${ }^{2}$ Assistant Professor, Department of Nursing, Dankook University, Cheonan \\ ${ }^{3}$ Associate Professor, College of Nursing, Yonsei University, Seoul, Korea
}

\begin{abstract}
Purpose: The purpose of this study was to identify factors influencing maternal identity of Korean primiparas. Methods: The data were collected by a self-report questionnaire in 2006. The participants were 210 healthy primiparous women who delivered at one of three medical centers and revisited the outpatient department for follow up between 4 to 6 weeks after childbirth. Data were analyzed using the SPSS WIN 17.0 program with descriptive statistics, t-test, one way ANOVA, Pearson correlation coefficient, and stepwise multiple regression. Results: Maternal identity was significantly correlated with Taekyo accomplishment, the culturally based prenatal preparation $(p<.001)$. Transitional gratification to motherhood $(p<.001)$, postpartum depression $(p<.001)$, childcare stress $(p<.001)$, infant temperament $(p<.001)$, and social support $(p<.001)$ were also significantly correlated with maternal identity. The stepwise multiple regression analysis showed that maternal identity was significantly predicted by transitional gratification to motherhood, infant temperament, childcare stress, and Taekyo accomplishment. These variables explained $31.0 \%$ of the variance of maternal identity. Conclusion: The results of this study suggest that postpartum nursing interventions to promote maternal identity should focus on reinforcing education and support for reducing childcare stress and infant difficulty, and increasing transitional gratification to motherhood. Also, prenatal encouragement and education for improving Taekyo accomplishment may be helpful to promote maternal identity after birth.
\end{abstract}

Key words: Primiparity, Maternal behavior, Maternal fetal relations, Pleasure, Identity

\section{INTRODUCTION}

Maternal identity refers to the perception and emotions as a mother in the maternal-infant relationship, which is formed through the process of psychosocial transition of becoming a mother after childbirth (Fontenot, 2007; Mercer, 2006). According to Rubin (1984), the core of maternal identity resides in the concepts of $I$ (mother) and you (infant), which influence each other. Maternal identity is formed through replication (mimicry and role play), fantasy, and dedifferentiation (introjections - projection - rejection) in the individual and repeated interrelations between mother and infant, and is expressed as positive attention for her baby and herself as a mother without any qualification. Prenatal acceptance of the fact that she will be a mother in the future is initially required, and maternal identity is enhanced and consolidated as she comes to know her infant(s) and what to expect of them after birth (Rubin).

When maternal identity is positively formed, the primiparous woman can feel a sense of stability as a mother and predict her baby's social ability and behavior patterns (Mercer \& Walker, 2006). However, confusion of maternal identity has been reported to delay assurance of becoming a mother, causes problems such the risk for ignoring or abusing the infants, and delay the infants' physical and social development (McGrath, Records, \& Rice, 2008; Mercer \& Walker).

Previous studies about factors influencing maternal identity reported that maternal identity was significantly correlated with childcare stress (Hassall, Rose, \& McDonald, 2005; Kowk \& Wong, 2000; Oh, 2001), infant temperament (Mantymaa, Puura, Luoma, Salmelin, \& Tamminen, 2006; Mercer, 2004), postpartum depression (Barr,

\footnotetext{
Address reprint requests to: Song, Ju-Eun

Department of Nursing, Dankook University, Anseo-dong, Dongnam-gu, Cheonan 330-714, Korea

Tel:+82-41-550-3987 Fax:+82-41-550-3888 E-mail: songje@dankook.ac.kr, songje72@hanmail.net

Received: May2,2011 Revised: May 23,2011 Accepted: December 19,2011
} 
2008; Fowles, 1998; Oh), and social support (Mercer, 2006; Warren, 2005). However, these studies reported individual relationships between maternal identity and some of these influencing factors, and lacked simultaneous analysis of these influencing factors as well as exploration of the relationship between maternal identity and transitional gratification to motherhood.

The transitional gratification to motherhood means the satisfaction, enjoyment, reward, and pleasure that a woman experiences in interactions with her infant and in fulfilling the tasks of the maternal role after the childbirth (Mercer, 2006). Gratification to mothering is an activator to preserve in the maternal role and identity (Logsdon, Wisner, \& Pinto-Foltz, 2006) and self-satisfaction as a mother is positively related with role acquisition and self-assessment as a mother (Fontenot, 2007; Mercer, 2004). Therefore, it is very important that positive emotion such as transitional gratification or pleasure to motherhood may have positive effect on the process of becoming a mother and maternal role performance. However, there is no study to verify the relationship between maternal identity which is base of the maternal role performance and transitional gratification to motherhood.

In addition, Korea has a unique prenatal folk belief and behavior system called Taekyo, which is a correction and control of behaviors and words to exert good influences on the fetus during pregnancy (Hong \& Lee, 2006). Taekyo is accomplished by the pregnant woman' s efforts to contribute to a healthy baby by focusing their mind and behaviors throughout the pregnancy. Therefore, Taekyo may have a positive effect on the pregnant women's psychological and emotional preparations for becoming a mother and may positively influence maternal identity of Korean postpartum women after childbirth. Yet, this has not been investigated.

The purpose of this study was to identify the influencing factors of maternal identity including not only the various previously identified factors such as postpartum depression, childcare stress, infant temperament, and social support, but also new possible factors such as transitional gratification to motherhood and Taekyo accomplishment using multiple regression analysis, which can be used to establish a prediction equation in which the independent variables are each assigned a weigh based on their relationship to the dependent variable (Munro \& Page, 1993). Verifying the significant predictors on maternal identity could promote a comprehensive understanding on the phenomenon of maternal identity in Korea and guide nursing interventions to promote positive maternal identity. To approach these aims, the main research question of this study was "What are the predictors of maternal identity of the primipara in Korea?"

\section{METHODS}

\section{Study design}

This study was a descriptive correlation and cross-sectional design using a self-reported questionnaire.

\section{Samples and setting}

A convenience sample of postpartum primiparous women was recruited from the outpatient department of one university hospital and two maternity centers in Kyunggi province, Korea. All participants were healthy primiparous women due for postpartum checkup between 4 to 6 weeks after childbirth. The inclusion criteria were primiparas who delivered after 37 weeks gestation, delivery of infant weighing over 2,500 gm, and agreement to participate in the research. Exclusion criteria were delivery of twins, or had an anemia (Hgb $<10.0 \mathrm{mg} / \mathrm{dL}$ ), thyroid disease, postpartum infection, or history of depression. From a total of 259 primiparous women who were invited to participate, 234 women voluntarily agreed to participate (response rate of $90.3 \%$ ), and 210 women completed the questionnaire without missing data.

\section{Instruments}

Data were collected using the well-established following questionnaires.

Maternal identity was measured using the Semantic Differential Scale-Myself as Mother (SD-self) developed by Walker (1977) based on the scale of Osgood, Suji, and Tannenbaum (1957) and translated into Korean by Koh (1996). This inventory consists of 11-items rated on a 7-point Likert scale (1 to 7) with higher scores indicating positive identity about self as a mother. The internal consistency reliability (Cronbach's alpha $=.81-.83)$ and content validity were established for use in the Korean postpartum women (Koh). In this study, the internal consistency reliability using Cronbach's alpha was .83 .

Taekyo accomplishment was measured using the revised subscale of the Taekyo Accomplishment Inventory developed by Choi and 
Kim (1995). Because many items were very similar to each other, the 19 original items were revised into 10 -items through pilot test for clarity. The 10-items were rated on a 5-point Likert scale (1 to 5) with higher scores indicating higher degree of Taekyo practice. Content validity was tested before use by three experts and internal consistency reliability was tested in a separated sample of 30 postpartum women visiting the outpatient department of one of the study hospitals (Cronbach's alpha =.88). In this study, the internal consistency reliability using Cronbach's alpha was .88 .

Transitional gratification to motherhood was measured using the Maternal Gratification Checklist developed by Russell (1974) and translated into Korean by Yee (1993). This instrument consists of 12items rated on a 4-point Likert scale (1 to 4) with higher scores indicating a higher degree of transitional gratification to motherhood. The internal consistency reliability (Cronbach's alpha $=.83$ ) and content validity were established for use in Korean postpartum women (Yee). In this study, the internal consistency reliability using Cronbach's alpha was 88 .

Postpartum depression was measured using the Edinburgh Postnatal Depression Scale (EPDS) developed by Cox, Holden, and Sagovsky (1987) and translated into Korean by Lee and Lee (2000). The EPDS consists of 10-items rated on a 4-point Likert scale (0 to 3 ) with higher scores indicating a higher degree of depression. The internal consistency reliability (Cronbach's alpha $=.79$ ) and content validity were established for use in Korean postpartum women (Lee \& Lee). This study also showed good internal consistency reliability (Cronbach's alpha $=.80$ ) for this instrument.

Childcare stress was measured using the Childcare Stress Inventory (CSI) developed by Cutrona (1984) and translated into Korean by Chun (1990). The CSI consists of 16-items rated on a 5-point Likert scale (1 to 5) with higher scores indicating a higher degree of childcare stress as perceived by the mother. The internal consistency reliability (Cronbach's alpha $=.87$ ) and content validity were established for use in Korean postpartum women (Kwon, 1997). In this study, the Cronbach's alpha was .85 .

Infant temperament was measured using the revised fussy-difficult subscale of the Infant Characteristics Questionnaire (ICQ) developed by Bates, Freeland, and Lounsbury (1979) and translated into Korean by Yee (1993). Of the nine items in the fussy-difficult subscale, four items were excluded as they were not applicable to infants under 3 months of age. The 5-items used for this study were rated on a 7-point
Likert scale (1 to 7) with higher scores indicating a more difficult temperament of the child. The internal consistency reliability of the modified scales was 0.77 in this study.

Social support was measured using the Social Support Inventory (SSI) developed by Lee (1992). This inventory consisted of 21-items rated on a 5-point Likert scale (1 to 5) with higher scores indicating a higher degree of social support as perceived by the mother. Internal consistency reliability (Cronbach's alpha $=.87$ ) and content validity were established for use in Korean postpartum women (Lee). This study also found high internal consistency reliability (Cronbach's al$\mathrm{pha}=.94)$.

Demographic characteristics such as age, family type, educational level, occupation, economic status, whether pregnancy was planned, delivery type, feeding method and baby's sex were also measured.

\section{Procedure}

After receiving of research approval from nursing department in the hospitals, participants were recruited from the waiting area in the outpatient department in 2006. After training of assessor about research purpose and data collection procedure, the researcher and two research assistants approached the waiting women and explained the purpose of the study, guarantees for anonymity and credibility, and expected time to complete a questionnaire (10 minutes). Written informed consent was obtained from all women who agreed to participate in the study, and small present was offered as an incentive. The questionnaire set was given to interested women and collected on the spot immediately after completion.

\section{Data analyses}

The data were analyzed with SPSS version 17.0 (SPSS, Chicago, IL, USA) using the following analyses using a $p$-value below .05 as level of significance. Descriptive statistics such as frequency, mean and standard deviation were used to describe the general characteristics and main study variables of this study. Independent sample t-test and one-way ANOVA were used to analyze the difference of maternal identity by general characteristics. Pearson correlation coefficients were calculated to identify the relationships between maternal identity and influencing variables. Stepwise multiple regression analysis was done to identify the influencing factors of maternal identity be- 
cause regression analysis is a useful technique that allows us to predict outcomes and explain the interrelationships among variables (Munro \& Page, 1993). In addition, multiple regression analysis is effectively used to establish a prediction equation in which the independent variables are each assigned a weigh based on their relationship to the dependent variable (Munro \& Page). Therefore, it is very effective multivariate analytic method to overcome the limitation of univariate analysis such as correlation, which cannot control the interrelationships among variables.

Before performing the multiple regression analysis, multicollinearity test was done to check whether the degree of interrelatedness of the independent variables in this study is problematic or not (Munro \& Page, 1993). As a result of multicollinearity test, the range of tolerance was verified as 0.55 to 0.89 (evaluation criteria is $>0.1$ ), variation inflation factor was 1.13 to 1.82 (evaluation criteria is $<10$ ) and condition index was 8.18 to 16.14 (evaluation criteria is $<30$ ). This analysis satisfied the suggested values of multicollinearity evaluation for regression analysis (Munro \& Page).

In regard to appropriateness of sample size, the following predictors were included for regression analysis: general characteristics significantly explained through univariate analysis as well as six independent variables, i.e., Taekyo accomplishment, transitional gratifica- tion to motherhood, postpartum depression, childcare stress, infant temperament, and social support. Because there was no general characteristic significantly explained through univariate analysis, the number of predictors was six. In consideration of the principle that at least 10 participants per predictor should be ensured to set a stable prediction equation in multiple regression analysis (Nunnally \& Bernstein, 1994), the sample size of this study was considered as sufficient for this analysis.

\section{RESULTS}

\section{General characteristics of the sample}

More than half of the participants were aged 30 to 39 years (54.3\%) and the majority were university graduate $(73.8 \%)$ living with their husbands only (85.5\%). More than half of the participants were fulltime housewives (53.8\%), of middle economic class (66.2\%), and noted a planned pregnancy (60.9\%). Most participants had delivered vaginally $(71.4 \%)$ and reported mixed feeding state (54.3\%). Slight more than half of the participants delivered a boy (51.9\%). There were no differences in maternal identity by general characteristics (Table 1).

Table 1. General Characteristics and Differences in Maternal Identity

\begin{tabular}{|c|c|c|c|c|c|}
\hline Characteristics & Categories & $n(\%)^{*}$ & Mean $\pm S D$ & tor $F$ & $p$ \\
\hline \multirow[t]{2}{*}{ Age (yr) } & $20-29$ & $96(45.7)$ & $52.0 \pm 7.42$ & 0.42 & .677 \\
\hline & $30-39$ & $114(54.3)$ & $51.5 \pm 8.21$ & & \\
\hline \multirow[t]{2}{*}{ Family type } & Nuclear & $180(85.7)$ & $51.8 \pm 7.96$ & 0.06 & .954 \\
\hline & Extended family & $30(14.3)$ & $51.7 \pm 7.26$ & & \\
\hline Educational level & High school & 55 (26.2) & $52.3 \pm 8.44$ & 0.56 & .575 \\
\hline \multirow[t]{2}{*}{ Occupation } & No & 113 (53.8) & $51.5 \pm 8.00$ & -0.65 & .516 \\
\hline & Yes & $96(45.7)$ & $52.2 \pm 7.65$ & & \\
\hline \multirow[t]{3}{*}{ Economic status } & Low & $32(15.2)$ & $51.0 \pm 7.74$ & 2.94 & .055 \\
\hline & Middle & $139(66.2)$ & $51.2 \pm 7.09$ & & \\
\hline & High & 39 (18.6) & $54.5 \pm 9.90$ & & \\
\hline Planned pregnancy & Yes & $128(60.9)$ & $52.2 \pm 7.84$ & -0.97 & .332 \\
\hline \multirow[t]{2}{*}{ Delivery type } & NSVD & $150(71.4)$ & $52.3 \pm 7.58$ & 1.59 & .112 \\
\hline & Cesarean & $60(28.6)$ & $50.4 \pm 8.39$ & & \\
\hline \multirow[t]{3}{*}{ Feeding method } & $\mathrm{BF}$ & $69(32.9)$ & $53.3 \pm 8.98$ & 2.10 & .126 \\
\hline & $\mathrm{MF}$ & $114(54.3)$ & $50.9 \pm 6.46$ & & \\
\hline & FF & $27(12.9)$ & $51.0 \pm 9.63$ & & \\
\hline \multirow[t]{2}{*}{ Baby's gender } & Male & $108(51.9)$ & $52.4 \pm 7.59$ & 1.11 & .268 \\
\hline & Female & $100(48.1)$ & $51.2 \pm 8.11$ & & \\
\hline
\end{tabular}

* Valid percent.

NSVD=Normal spontaneous vaginal delivery; BF=Breast feeding; MF=Mixed feeding; FF=Formula feeding. 


\section{Maternal identity and independent variables}

The mean score of maternal identity was 51.7 (SD 7.85). Those of Taekyo accomplishment and transitional gratification to motherhood were 36.9 (SD 6.45) and 35.2 (SD 5.82), respectively. The mean score of postpartum depression was 8.74 (SD 4.49) and 45 participants (21.4\%) were diagnosed with depression considering cut point of depression diagnosis ( $\geq 13$ points). Those of childcare stress, infant temperament, and perceived social support were 44.2 (SD 9.83), 21.2 (SD 4.89), and 73.6 (SD 9.71), respectively (Table 2).

\section{Correlations among the study variables}

All of the correlations between maternal identity and the independent variables were significant and in the anticipated direction. Positive correlations were found between maternal identity and Taekyo accomplishment $(\mathrm{r}=.30, p<.001)$, transitional gratification to motherhood $(\mathrm{r}=.44, p<.001)$, and social support $(\mathrm{r}=.29, p<.001)$. Negative correlations were noted between maternal identity and postpartum depression $(\mathrm{r}=-.32, p<.001)$, childcare stress $(\mathrm{r}=-.46, p<.001)$, and infant temperament $(\mathrm{r}=-.35, p<.001)$. In addition, all correlations among study variables were in the anticipated direction and indicate that most variables appeared to be statistically related to each other (Table 3).

\section{Predictors on the maternal identity in the Primiparas}

Because the multicollinearity evaluation for regression analysis was satisfied the suggested values, stepwise multiple regression analysis was used to examine simultaneously the relation of several predic-

Table 2. Descriptive Statistics of the Study Variables

$(N=210)$

\begin{tabular}{lrrrr}
\hline Variables & $\begin{array}{c}\text { Possible } \\
\text { range }\end{array}$ & Mean \pm SD & Min. Max. \\
\hline $\begin{array}{l}\text { Dependent variable } \\
\quad \text { Maternal identity }\end{array}$ & $11-77$ & $51.7 \pm 7.85$ & 22 & 74 \\
Independent variables & & & & \\
$\quad$ Taekyo accomplishment & $10-50$ & $36.9 \pm 6.45$ & 20 & 50 \\
Transitional gratification to motherhood & $12-48$ & $35.2 \pm 5.82$ & 14 & 48 \\
Postpartum depression & $0-30$ & $8.74 \pm 4.49$ & 1 & 23 \\
Childcare stress & $16-80$ & $44.2 \pm 9.83$ & 16 & 71 \\
Infant characteristics & $5-35$ & $21.2 \pm 4.89$ & 5 & 34 \\
Social support & $21-84$ & $73.6 \pm 9.71$ & 38 & 84 \\
\hline
\end{tabular}

Min. $=$ Minimum; Max. $=$ Maximum . tors to the dependent variable of maternal identity. Six independent variables that were correlated or associated with the maternal identity in the univariate analysis were put into a multiple regression analysis. Among these six independent variables, four variables were included in the regression model, and maternal identity was significantly predicted by transitional gratification to motherhood $(\beta=.28)$, infant temperament $(\beta=-.24)$, childcare stress $(\beta=-.16)$, and Taekyo accomplishment $(\beta=.15)$. The first time mother who had higher score of transitional gratification to motherhood $(\beta=.28, p<.001)$ and Taegyo accomplishment during the pregnancy $(\beta=.15, p=.013)$, lower score of infant difficult temperament $(\beta=-.24, p=.001)$ and childcare stress $(\beta=-.16, p=.035)$ had higher score of maternal identity. The overall F score of multiple regression model was highly significant $(\mathrm{F}=24.80, p<.001)$ and $31.3 \%$ of variance of maternal identity was explained by these four predictors in the Korean primiparous women (Table 4).

\section{DISCUSSION}

\section{Maternal identity of Korean Primiparas}

The mean score of maternal identity in primiparas within 4 to 6 weeks after childbirth was $51.7 \pm 7.85$. This score was similar with other Korean studies, which reported 51.4 (Koh, 1996) and 54.5 (Chae \& Kim, 2011), but was lower than the score of 66.7 in sample of 64 healthy primiparas in the 4 to 6 weeks postpartum (Walker, Crain, \& Thompson, 1986) and the score of 64.8 in sample of 168 primiparas

Table 3. Correlation Coefficients among Study Variables $\quad(N=210)$

\begin{tabular}{lcccccc}
\hline Study & MI & TA & TGM & PD & CS & IT \\
variables & $r$ & $r$ & $r$ & $r$ & $r$ & $r$ \\
& $(p)$ & $(p)$ & $(p)$ & $(p)$ & $(p)$ & $(p)$ \\
\hline TA & .30 & 1 & & & & \\
TGM & $(<.001)$ & & & & & \\
& .44 & .31 & 1 & & & \\
PD & $(<.001)$ & $(<.001)$ & & & & \\
& -.32 & -.19 & -.40 & 1 & & \\
CS & $(<.001)$ & $(.005)$ & $(<.001)$ & & & \\
& -.46 & -.25 & -.49 & .49 & 1 & \\
IT & $(<.001)$ & $(<.001)$ & $(<.001)$ & $(<.001)$ & & \\
& -.35 & -.05 & -.10 & .14 & .49 & 1 \\
SS & $(<.001)$ & $(.450)$ & $(.131)$ & $(.041)$ & $(<.001)$ & \\
& .29 & .36 & .37 & -.38 & -.26 & .02 \\
& $(<.001)$ & $(<.001)$ & $(<.001)$ & $(<.001)$ & $(<.001)$ & $(.742)$ \\
\hline
\end{tabular}

TA=Taekyo accomplishment; TGM=Transitional gratification to motherhood; $\mathrm{PD}=$ Postpartum depression; CS = Childcare stress; $\mathrm{IT}=$ Infant temperament; SS=Social support. 
Table 4. Predicting Factors of Maternal Identity

\begin{tabular}{lcccccc}
\hline Factors & $\mathrm{B}$ & Std. Error & $\beta$ & $\mathrm{T}(\mathrm{p})$ & $\mathrm{F}(\mathrm{p})$ & Adjusted $\mathrm{R}^{2}$ \\
\hline Constant & 45.27 & 5.30 & & & & \\
Transitional gratification to motherhood & 0.38 & 0.09 & .28 & $4.09(<.001)$ & \\
Infant temperament & -0.38 & 0.11 & -.24 & $-3.51(.001)$ & $24.80(<.001)$ & .31 \\
Childcare stress & -0.13 & 0.06 & -.16 & $-2.12(.035)$ & \\
Taekyo accomplishment & 0.19 & 0.07 & .15 & $2.51(.013)$ & \\
\hline
\end{tabular}

after 8 weeks postpartum in the United States (Fowles, 1998). This finding implies that maternal identity may be related to the culture or social value and supports the opinion that maternal identity of Korean primiparous women is lower than that of other countries' primiparous women (Lee, 1992). To verify the cultural difference and transitional process of becoming a mother in different cultures, comparative studies between cultures are needed considering cultural or social values related to motherhood. Another possible reason for the lower score in this study, as compared to the prior studies (Fowles; Walker et al.) may be related to translation of the SD-self instrument into Korean. Total score of maternal identity was measured and summed by an 11-item, 7-point semantic differential scale consisting of bipolar adjective pairs. A minor difference in the translation from English to Korean, and social directivity contained in adjective pairs of scale may have lead to the score difference. Further studies would be helpful to identity the properties of the instrument and the validity of the Korean SD-self instrument.

Walker et al. (1986) reported that mothers' attitudes about themselves became more positive from 1 to 3 days to 4 to 6 weeks postpartum. However, some Korean studies reported that maternal identity decreases with time (Chae \& Kim, 2011; Oh, 2001). In detail, Chae and Kim reported that maternal identity of 3 to 4 weeks postpartum was lower than that immediately after childbirth and Oh reported that maternal identity at 8 weeks was lower than that at 4 weeks postpartum. Considering that maternal identity forms and develops with the passage of time, prospective studies are also necessary to identify and compare the changing pattern of maternal identity between different cultures.

\section{Predictors of maternal identity}

In this study, multiple regression analysis was used to establish a prediction equation because this method was thought to be effective multivariate analytic method to overcome the limitation of univari- ate analysis (Munro \& Page, 1993) which cannot control the interrelationships among variables. As a result, the regression model including transitional gratification to motherhood, infant temperament, childcare stress, and Taegyo accomplishment was found to be significant $(\mathrm{F}=24.80, p<.001)$. Therefore, the detail discussion about each predictor of maternal identity will be described in the following.

First, we found that transitional gratification to motherhood was a strong explaining factor on maternal identity, which is very meaningful in that this is the first work to explore the influences of the transitional gratification to motherhood on maternal identity. Although primiparous women may experience various difficulties and confusion while adapting to their new roles as a mother, many find gratification from mothering (Logsdon et al., 2006). Women who perceived a higher level of stress tend to feel less satisfied in taking up the maternal role and feel a role-related conflict (Hassall et al., 2005). If primiparous women are conflicted about their role without experiencing self-satisfaction or gratification as mother, it may cause confusion in the formation of maternal identity (Logsdon et al.). This study suggests that measures are needed to help women in identifying positive feeling and improving self-evaluation through continuous support and education.

Second, infant temperament was another significant influencing factor on maternal identity in this study, which is in agreement with results of prior studies on primiparous mothers (Mantymaa et al., 2006; Mercer, 2004). Caring for infants with difficult temperament has been noted as an obstacle to perceived childcare ability with possible dissatisfaction of a woman's ability as a mother (Mercer). Furthermore, mothers with infants who frequently cry reported feelings of unease, anxiety, depression and increased stress (McGrath et al., 2008), which may interrupt the formation of positive maternal identity. Accordingly, it is necessary not only to teach infant care skills, such as bathing and breast-feeding, but also to provide education that may promote understanding of infant behavior or temperamental characteristics for enhancing positive maternal identity in primiparas. 
Third, childcare stress was also verified as an important predictor of maternal identity. Although there is little empirical data to directly explain the relationship between childcare stress and maternal identity, this finding is in agreement with results of prior studies that noted a negative relationship between childcare stress and maternal role competence or satisfaction, which can be considered as a similar concept to maternal identity (Hassall et al., 2005; Kowk \& Wong, 2000). Postpartum women experience various physical and psychological changes following childbirth while adjusting to the responsibility of taking care of the newborn which is completely dependent on them. Childcare stress has been noted as the most important and frequently reported problem (Hassall et al.), particularly in primiparous women due to lack of knowledge of childcare. This study underscores the importance of better preparing first-time mothers to undertake newborn care as a buffer on childcare stress to eventually enhance the formation of positive maternal identity.

Lastly, the cultural concept of Taekyo accomplishment during pregnancy was presently revealed to be a significant influencing factor on maternal identity and may be a unique window of opportunity for prenatal influence. While prenatal care in Western society mainly focuses on maternal health indices and fetal physical development according to gestational weeks, Taekyo in Korea focuses on the behaviors and minds of pregnant women to provide positive influences to the fetus. As pregnant women are encouraged to use prudence in speech and action in order to ensure a physically and mentally healthy baby with excellent personality throughout the pregnancy, Taekyo is a more inclusive concept than the usual prenatal care. Taekyo has received little scholarly attention from Western medical science until recently, but the importance of Taekyo is now becoming more widely recognized. Maternal identity begins to form during pregnancy and is established and further developed during the postpartum period. Previous studies have shown that Taekyo accomplishment during pregnancy increases the maternal identity of pregnant women and promotes the attachment between a pregnant woman and her fetus (Chang, Kim, \& Kim, 2000). Hong and Lee's (2006) study of Ten-week and four-week Taekyo program, respectively, also reported increased maternal identity of pregnant women and implied the possibility of enhanced maternal identity during the postpartum period. However, it is true that while Korean pregnant women have a high level of interest in Taekyo, the actual practice of Taekyo is much less (Choi \& Kim, 1995). Furthermore, healthcare providers also lack interest in
Taekyo in prenatal care contact, despite acknowledging its importance (Choi \& Kim). Therefore, it is important to consider prenatal education integrated with Taekyo principles in detail and to support programs that encourage Taekyo practice during pregnancy, which may enhance positive maternal identity.

Although Mercer (2006) and Warren (2005) explained that the higher level of social support was significantly affect the higher level of maternal identity, social support was not included in the regression model of maternal identity in this study. This incongruence with previous studies may be related to lack of inclusion of childcare stress or infant temperament in prior studies. In other words, social support may affect childcare stress or mother's perception of infant temperament which are preceding variables of maternal identity. Therefore, it may have an indirect effect on maternal identity via childcare stress or infant temperament rather than directly affect on maternal identity. Actually, prior studies explained that social support have a positive effect on reducing of the childcare stress of mother (Kim et al., 2005) and perception of infant temperament by mother (Lee, Long, \& Boore, 2009). Therefore, further researches are needed to identify these relationships among independent variables through structural equation modeling or path analysis, in especially indirect effect of social support on maternal identity via childcare stress and perceived infant characteristics.

In addition, Barr (2008) and Oh (2001) reported that the negative relationship between postpartum depression and maternal identity, but postpartum depression was not also included in the regression model as a significant predictor. This incongruence with previous studies may be related to the following two reasons. First, Oh measured the postpartum depression using the postpartum depression scale developed by Bae's instrument (2004) for Korean women not EPDS developed by Cox et al. (1987), and Barr investigated the postpartum women who diagnosed as postpartum depression. These differences of the instrument $(\mathrm{Oh})$ and participants (Barr) may be related to the incongruent results. Second, it may be related to the multivariate analysis. In other words, postpartum depression is significantly related to the maternal identity in the univariate analysis which cannot control the interrelationships among independent variables and not considered a weigh based on their relationship to the dependent variable. Therefore, when multivariate analysis performed, more important or weigh independent variable were selected to the predictor of maternal identity. Therefore, replication studies are also needed 
to verify the true relationship between postpartum depression and maternal identity in the future.

This study was restricted in explaining casual relationships among the variables since the analysis was conducted through cross-sectional data collection and participants were selected by convenience sampling, requiring careful interpretation of generalizing the study results to all primiparous women. Also, Taegyo accomplishment during pregnancy was measured by memory recall, so it may have been insufficiently measured. Nevertheless, this is the first work to verify the affecting variables on maternal identity, which included not only already known variables (e.g. childcare stress and infant temperament) but also newly verified factors such as maternal gratification to motherhood and Taekyo accomplishment. Also, it is explained the relationship between maternal identity and cultural factor, Taegyo accomplishment during pregnancy in Korea.

\section{CONCLUSION}

The results of this study show that maternal identity in the new mothers is significantly predicted by transitional gratification to motherhood, infant temperament, childcare stress, and Taekyo accomplishment. From these results, it is concluded that nursing interventions for promoting maternal identity should focus on the education and support for reducing childcare stress, and promotion of understanding of infant temperament and transitional gratification to motherhood. Moreover, the study results suggest the possibility that interventions for promoting and activating Taekyo accomplishment in Korea could promote maternal identity after childbirth. Further studies are needed to develop such interventions and verify their effects. Also, future research may benefit from repeated studies on primiparous women of various cultural background as well as comparing differences between primiparas and multiparas.

\section{REFERENCES}

Bae, J. Y. (2002). Construction of postpartum depression model. Journal of Korean Academy of Psychiatric and Mental Health Nursing, 11, 572-587.

Barr, J. A. (2008). Postpartum depression, delayed maternal adaptation, and mechanical infant caring: A phenomenological hermeneutic study. International Journal of Nursing Studies, 45, 362-369. doi: 10.1016/j.ijnurstu.2006.10.002

Chae, H. J., \& Kim, S. (2011). Effects of maternal role practice education on becoming a mother. Korean Journal of Women Health Nursing, 17, 52 63. doi: 10.4069/kjwhn.2011.17.1.52

Chang, S. B., Kim, K. Y., \& Kim, E. S. (2000). Changes of maternal-infant attachment and self efficacy for delivery after the Taekyo-perspective prenatal class. Korean Journal of Women Health Nursing, 7, 7-17.

Choi, Y. S., \& Kim, H. O. (1995). A survey on the practice of Taekyo among childbearing couples. Korean Journal of Women Health Nursing, 1, 153173.

Chun, C. A. (1990). The effect of postpartum stress and social network on the postpartum depression. Unpublished master's thesis, Korea University, Seoul.

Fontenot, H. B. (2007). Transition and adaptation to adoptive motherhood. Journal of Obstetric, Gynecologic, and Neonatal Nursing, 36, 175-182. doi: 10.1111/j.1552-6909.2007.00134.x

Fowles, E. R. (1998). The relationship between maternal role attainment and postpartum depression. Health Care for Women International, 19, 83-94.

Hassall, R., Rose, J., \& McDonald, J. (2005). Parenting stress in mothers of children with an intellectual disability: The effects of parental cognitions in relation to child characteristics and family support. Journal of Intellectual Disability Research, 49, 405-418.

Hong, E. J., \& Lee, J. Y. (2006). The effects of the 'Taegyo' program using art therapy techniques in the anxiety, postpartum depression and maternal identity of pregnant women. The Korean Journal of Human Development, 13(1), 97-115.

Kim, H. S., Oh, K. S., Shin, Y. H., Kim, T. I., Yoo, H. N., Sim, M. K., et al. (2005). Factors influencing parenting stress in primiparas. Journal of Korean Academy of Child Health Nursing, 11, 290-300.

Koh, H. J. (1996). The relationship between role strain and identity in first-time mothers with regard to employment status. Unpublished doctoral dissertation, Ewha Womans University, Seoul.

Kowk, S., \& Wong, D. (2000). Mental health of parents with young children in Hong Kong: The roles of parenting stress and parenting self-efficacy. Child and Family Social Work, 5, 57-65.

Kwon, J. H. (1997). A test of a vulnerability-stress model of postpartum depression. Korean Journal of Clinical Psychology, 16(2), 55-66.

Lee, E. S. (1992). Maternal role attainment of primiparous during the postpartum period. Korean Journal of Women Health Nursing, 2, 5-20.

Lee, S. N., Long, A., \& Boore, J. (2009). Taiwanese women's experience of becoming a mother to a very-low-birth-weight preterm infant: A grounded theory study. International Journal of Nursing Studies, 46, 326-336.

Lee, T. G., \& Lee, J. H. (2000). Literature review of postpartum depression. The Journal of Oriental Gynecology, 13(1), 518-530.

Logsdon, M. C., Wisner, K. L., \& Pinto-Foltz, M. D. (2006). The impact of postpartum depression on mothering. Journal of Obstetric, Gynecologic, and Neonatal Nursing, 35, 652-658. doi: 10.1111/J.1552-6909.2006. 00087.x

Mantymaa, M., Puura, K., Luoma, I., Salmelin, R. K., \& Tamminen, T. (2006). Mother's early perception of her infant's difficult temperament, parenting stress and early mother-infant interaction. Nordic Journal of Psychiatry, 60, 379-386. doi: 10.1080/08039480600937280

McGrath, J. M., Records, K., \& Rice, M. (2008). Maternal depression and infant temperament characteristics. Infant Behavior \& Development, 31, 71-80. doi: 10.1016/j.infbeh.2007.07.001 
Mercer, R. T. (2004). Becoming a mother versus maternal role attainment. Journal of Nursing Scholarship, 36, 226-232. doi: 10.1111/j.1547-5069. 2004.04042.x

Mercer, R. T. (2006). Nursing support of the process of becoming a mother. Journal of Obstetric, Gynecologic, and Neonatal Nursing, 35, 649-651. doi: 10.1111/j.1552-6909.2006.00086.x

Mercer, R. T., \& Walker, L. O. (2006). A review of nursing interventions to foster becoming a mother. Journal of Obstetric, Gynecologic, and Neonatal Nursing, 35, 568-582. doi: 10.1111/j.1552-6909.2006.00080.x

Munro, B. H., \& Page, E. B. (1993). Statistical methods for health care research (2nd ed.). Philadelpia: J.B. Lippincott, USA.

Nunnally, J. C., \& Bernstein, I. H. (1994). Psychometric theory (3rd ed.). New York: McGraw-Hill, USA.
Oh, H. E. (2001). The relationship among maternal identity, role attainment and postpartum depression. Journal of the Korean Society of Maternal and Child Health, 5, 255-266.

Rubin, R. (1984). Maternal identity and the maternal experience. New York: Springer.

Walker, L. O., Crain, H., \& Thompson, E. (1986). Maternal role attainment and identity in the postpartum period: Stability and change. Nursing Research, 35, 68-71.

Warren, P. L. (2005). First time mother: Social support and confidence in infant care. Journal of Advanced Nursing, 50, 479-488. doi: 10.1111/ j.1365-2648.2005.03425.x

Yee, Y. H. (1993). Patterns of infant-father attachment in the strange situation. Journal of Korean Home Management Association, 11(2), 40-54. 